This item was submitted to Loughborough's Research Repository by the author.

Items in Figshare are protected by copyright, with all rights reserved, unless otherwise indicated.

\title{
An approach for controller fault detection
}

PLEASE CITE THE PUBLISHED VERSION

PUBLISHER

(C) IEEE

VERSION

VoR (Version of Record)

LICENCE

CC BY-NC-ND 4.0

\section{REPOSITORY RECORD}

Dai, Chengwei, Shuang-Hua Yang, and Liansheng Tan. 2019. "An Approach for Controller Fault Detection". figshare. https://hdl.handle.net/2134/4127. 
This item was submitted to Loughborough's Institutional Repository (https://dspace.lboro.ac.uk/) by the author and is made available under the following Creative Commons Licence conditions.

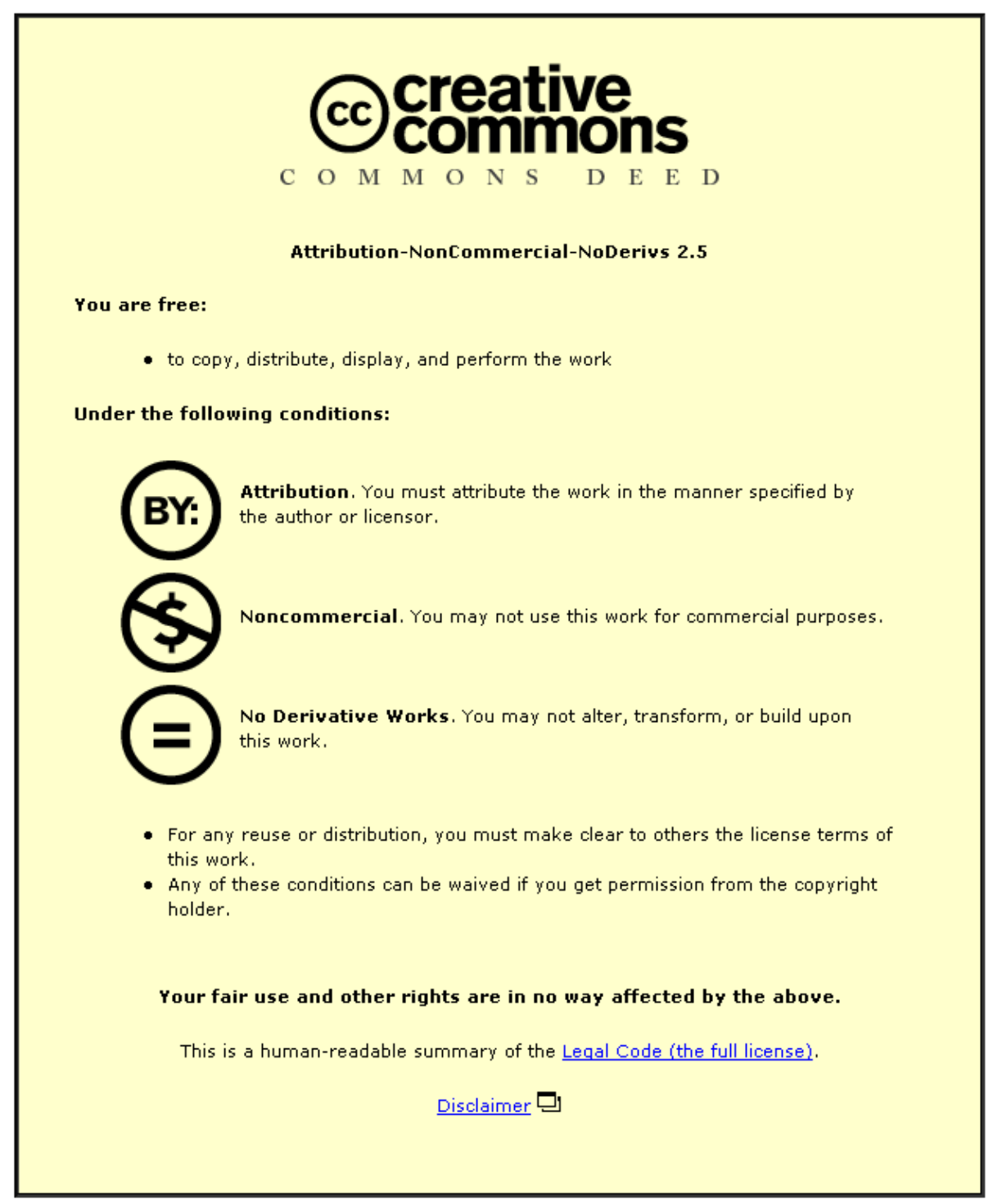

For the full text of this licence, please go to: http://creativecommons.org/licenses/by-nc-nd/2.5/ 


\section{An Approach for Controller Fault Detection*}

\author{
C. Dai, S. H. Yang \\ Department of Computer Science \\ Loughborough University \\ Loughborough, Leicestershire LE11 3TUUK \\ \{C.Dai , S.H.Yang\}@ lboro.ac.uk
}

\author{
Liansheng Tan \\ Department of Computer Science \\ Central China Normal University \\ Wuhan, China, 430079. \\ L.Tan@ccnu.edu.cn
}

\begin{abstract}
Monitoring and maintaining of control software becomes more and more important and difficult with the increase of control software in size and complexity. In this paper, an approach for control software fault detection is proposed, which is based on the monitoring of the discrepancies between the control outputs of the actual controller and the benchmark controller, a Linear Quadratic Gaussian (LQG) controller. The discrepancies are assumed to be Gaussian distribution with a stable mean under the normal situation. Faults in the actual controller are characterized by sudden jumps in the mean of the discrepancies. The fault detection is transferred into a jump point identification problem. A detector based on Generalized Likelihood Ratio (GLR) test is employed for the jump point identification. The proposed approach is applicable to general control software even it is only illustrated through a water heater case study with a simple PID controller.

Index Terms - Controller Fault Detection; Linear Quadratic Gaussian (LQG); Generalized Likelihood Ratio (GLR); Gaussian Distribution.
\end{abstract}

\section{INTRODUCTION}

With the wide incorporating advanced control strategies into computer based control systems, controller software becomes more and more complex. The steadily growing size and complexity of control software are making it extremely difficult to exhaustively test. It has shown that software complexity is a determinant factor leading to error-proneness of software [1]. Due to controller's crucial role in the control systems, even minor controller faults can lead to serious and expensive damages. The damages may range from those affecting the qualities of final products to those involving dangers to property or loss of human life. Early warning on emerging problems can provide invaluable benefits with appropriate actions taken to avoid serious process upsets. Therefore, it is an important issue in control management systems to detect the control software faults and raise alarms as early as the faults occur.

The main difficulty of the detection is the lack of a generally accepted definition of what constitutes desired behaviors of the black-box controllers. The desired behaviors will be adopted as the benchmark in the detection procedure to monitor the observed behaviors of the actual controller. In the traditional machine faults detection methods [2,3], the desired behaviors are described by an explicit mathematical model, which is identified from routine operation data. However, it is hard to establish an accurate mathematical model in practice for control software to cover all the possible situations due to process dynamics, noise and so on. In the software monitoring [4], the desired behaviors are formally specified, however nondeterminisms arise from specification languages and monitoring such nondeterminism specified system has to consider all possible legal behavior alternatives in order to avoid erroneous failure reports. Especially when the target is complex, the alternatives may exponentially increase.

This work introduces a novel way to detect control software faults. A LQG controller is designed here as the benchmark or a reference model for the target control software. Discrepancies between the benchmark and actual controller outputs are shown to be in an approximate Gaussian distribution [6]. A baseline of controller normal operation is treated as the asymptotic distribution of the discrepancies. Fault detection characterized by sudden jumps in the mean of the discrepancy is formulated as an online change point identification problem. A detector based on the GLR test is employed to online monitor the change point. The approach is illustrated through a water heater case study with a PID controller even it is applicable to general control software.

The rest of this paper is arranged as follows. A background introduction of LQG controller design is given in Section 2, followed by the fault detection based on the GLR test to monitor the behaviors of target controller in Section 3. Section 4 contains a case study, in which the proposed approach is employed in a water heater process. Section 5 gives the conclusions.

\section{LQG CONTROLLER AS A BENCHMARK}

The LQG control is a modern state-space technique for designing optimal dynamic regulators. It can be used to solve a family of regulator design problems in which the state is accessible.

Consider a system can be described as follows:

$\left\{\begin{array}{l}\dot{\mathbf{x}}=A \mathbf{x}+B \mathbf{u}+\mathbf{w}_{\text {proc }} \\ \mathbf{y}=C \mathbf{x}+\mathbf{v}_{\text {sensor }}\end{array}\right.$

The vector $\mathbf{u}$ represents process input or manipulated variables; the vector $\mathbf{y}$ describes process output measurements; the vector $X$ represents process state; the process noise $\mathbf{w}_{\text {proc }}$ and the measurement noise $\mathbf{v}_{\text {sensor }}$ are

\footnotetext{
This study was financially supported through a PhD studentship from Faculty of Science, Loughborough University, UK.
} 
independent and have constant power spectral density matrices $\mathbf{W}$ and $\mathbf{V}$ respectively.

The first step in the LQG controller design is to seek a state-feedback law that minimizes the cost function of regulation performance, which is measured by a quadratic performance criterion with user-specified weighting matrices in order to define the tradeoff between regulation performance and control effort.

$$
J=\lim _{t \rightarrow \infty} E\left[x(t)^{T} \mathbf{Q} x(t)+u(t)^{T} \mathbf{R} u(t)\right]
$$

where $\mathbf{Q}$ and $R$ are positive semidefinite weight matrices. The first term penalizes the deviation of $\mathbf{x}$ from zero, and the second term represents the cost of using the control actions.

Standard assumptions of the LQG problem are that the plant is controllable and observable, a positive weight is used for the control signal $R>0$ and the sensor noise satisfies $V>0$. With these assumptions in force, there is a unique controller $K$ that minimizes the LQG objective. The LQG controller consists of an optimal state-feedback gain and a state estimator; the optimal state-feedback and estimator gains, $K_{f}$ and $K_{e s t}$, can be determined by solving two algebraic Riccati equations as follows. The state-feedback gain $K_{f}$ is given by

$K_{f}=R^{-1} B^{T} S_{c}$

which $S_{c}$ is obtained from the solution of the Riccati equation

$A^{T} S_{c}+S_{c} A-S_{c} B R^{-1} S_{c}+Q=0$

The estimator gain is given by

$$
K_{e s t}=S_{d} C^{T} V^{-1}
$$

where $S_{d}$ is the unique positive definite solution of Equation 6.

$$
A S_{d}+S_{d} A^{T}-S_{d} C^{T} V^{-1} C S_{d}+W=0
$$

The LQG-optimal controller $K$ is thus

$$
K(s)=-K_{f}\left(s I-A+B K_{f}+K_{e s t} C\right)^{-1} K_{e s t} \text {, }
$$

where $s$ is the Laplace transfer symbol.

In addition to the above solution, there are other solutions for the LQG problem. The minimization of the LQG objective function yields a time-invariant optimal control law; therefore the LQG problem can be also solved via the infinite generalized predictive control (GPC) solution. Another way to solve the LQG problem is with the input-output transfer function using the approach of spectral factorisation and the solution of the Diophantine Identify [5].

The infinite prediction horizon of the $L Q R$ algorithm endows the algorithm with powerful stabilizing properties; it is shown that the LQR controller is stable for any reasonable linear plant as long as the objective function weight matrices $Q$ and $R$ are positive definite [5].

\section{FAult Detection}

The difference of control signals between the LQG and the actual controller is used to detect the faults if they occur. Theoretically, if the actual controller is in a healthy work state and tuned well, the outputs of the actual controller and the LQG controller will be very close and any fault occurring in the system will make the differences or residuals vary significantly. In practice, however, the residuals should significantly deviate from zero due to the miss-tuning of parameters in the actual controller, and measurement noise. One of the challenges in the fault detection is how to distinguish the fault from the noise in the residuals. Traditional detection systems simply compare the residuals against a threshold to determine if a fault has occurred. This requires carefully designed, well-behaved residuals, which often require extensive modeling and high computational efforts.

In this work, according to characteristics of the statistical distribution of the residuals, a detector based on the GLR test is employed to capture the fault information from the mixed residuals and raise an alarm as early as possible.

Consider the observed residual $r(t)$

$\left\{\begin{array}{l}r(t)=u_{a c t}(t)-u_{L Q G}(t)+r_{0}(t) \\ =r_{1}(t)+r_{0}(t)\end{array}\right.$

$r_{0}(t)$ is the white noise following Gaussian distribution with zero mean, $r_{1}(t)$ is the difference between the control signals of the LQG controller, $u_{L Q G}$, and the actual controller $u_{\text {act }}$. For a stable control process, it can be shown under general assumptions that the distribution of independent random residual $r_{1}(t)$ takes on a Gaussian distribution as the number of observations becomes large [6]. Therefore, $r(t)$ can be assumed to approximately follow the Gaussian distribution with the mean value $\mu$ and variance $\sigma$.

$$
p(r(t))=\frac{1}{\sqrt{2 \pi \sigma^{2}}} \exp \left(\frac{-(r(t)-\mu)^{2}}{2 \sigma^{2}}\right)
$$

where $p($.$) denotes the density function of the residual r(t)$. Collect a set of observed residual $r(k)$, where $\mathrm{k}$ is the number of sample data. Before the instant $t_{1}, r(k)$ follows Gaussian distribution, with $\mu_{0}$ mean and $\sigma_{0}$ variance.

Suppose a fault occurs at the instant $t_{1}$, the new generated residuals shift to a new distribution with a different mean denote by $\mu_{1}$; therefore, the early fault detection can be transferred into the identification of the change in the mean value $\mu$ (from $\mu_{0}$ to $\mu_{1}$ ). Based on the GLR test, a detector can be designed to detect the mean change through on-line monitoring. The decision rule of the detector is computed to test between the two following hypotheses about the parameter $\mu$. 
$\left\{\begin{array}{l}H_{0}: \mu=\mu_{0} \text { normal work state } \\ H_{1}: \mu=\mu_{1} \text { fault work state }\end{array}\right.$

As long as the decision is taken in favor of $H_{0}$, the sampling and test continue. The decision is taken in favor of $H_{1}$ once the fault occurs.

The decision rule is given by:

$$
s_{k}=\ln \frac{p\left(r(k) \mid \mu_{1}\right)}{p\left(r(k) \mid \mu_{0}\right)}
$$

where $s_{k}$ is the likelihood ratio.

For the fault case, it is hard to obtain an unbiased of the postchange distribution $p\left(r(t) \mid \mu_{1}\right)$. However, such accurate estimation is not crucial, the mean of the observed data in the faulty work state, $\mu_{1}$, has a significant difference from $\mu_{0}$ and this difference provides enough information to detect the faults [7] so we can assume the post-change distribution is still Gaussian. $\mu_{1}$ is calculated by maximum likelihood estimator.

$$
\mu_{1}=\frac{\sum_{i=1}^{k} r(i)}{k}
$$

Based on the above assumption, the decision rule can take a simple form, given by

$$
s_{k}=\frac{\mu_{1}-\mu_{0}}{\sigma_{0}{ }^{2}}\left(r(k)-\frac{\mu_{0}+\mu_{1}}{2}\right)
$$

The fault is identified using a threshold $\lambda$ as follows.

$s_{k}>\lambda: H_{1}$ is assumed i.e. a fault is detected

$s_{k} \leq \lambda: H_{0}$ is assumed i.e. no fault is detected

The principle of the controller fault detection is shown in Figure 1, which consists of two stages: the residual generation stage and the residual evaluation stage. In the residual generation stage, the benchmark controller, LQG, is designed to run in parallel to the actual controller. The LQG controller can be treated as a relative measure of the actual controller behavior. The residuals are generated from the differences between the control signals of the actual controller and the LQG controller.

The residual evaluation collects the residuals and generates the detection decision. It consists of three parts, a threshold, a maximum likelihood estimator and a fault detector. The maximum likelihood estimator is to online compute the mean of the moving residuals and sends the result to the fault detector. Based on the updating mean, the detector tests the observed residual data by comparing whether or not the likelihood ratio is within the predefined threshold range. Once the observed residuals are out of the range, an alarm will raise to show the monitored controller is in a faulty work state.

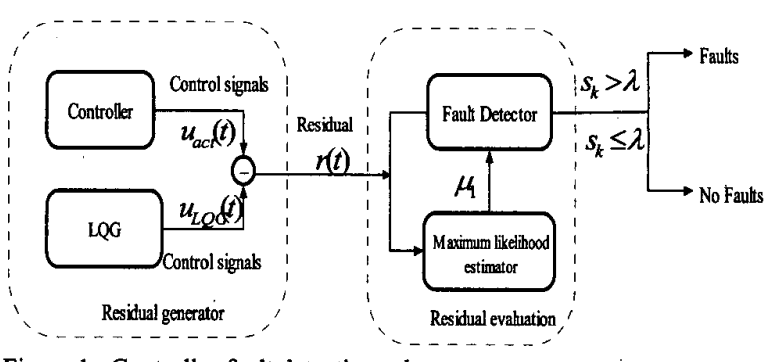

Figure 1: Controller fault detection scheme

\section{IV.A CASE STUDY}

In order to illustrate and evaluate how the proposed approach monitors the behaviors of target controllers, a water heater process has been used as a case study.

\section{A. Water Heater Process}

The water heater process is shown Figure 2. In this case study, the water temperature in the pipe is controlled by adjusting the flow of the stream. A PID controller is designed here to regulate the flow rate of stream to maintain a desired water temperature. The transfer function of the water heater process is $\frac{0.2965}{s+0.1802}$, where $s$ is the Laplace transfer symbol.

A sine wave noise and a white noise with zero mean are adopted in the water heater process as the process noise, $w_{\text {proc }}$, and the measurement noise, $v_{\text {sensor }}$, respectively.

Their noise intensities are given respectively by

$$
\left\{\begin{array}{l}
E\left(\mathbf{v}^{T} \mathbf{v}\right)=0.005 \\
E\left(\mathbf{w}^{T} \mathbf{w}\right)=0.998
\end{array}\right.
$$

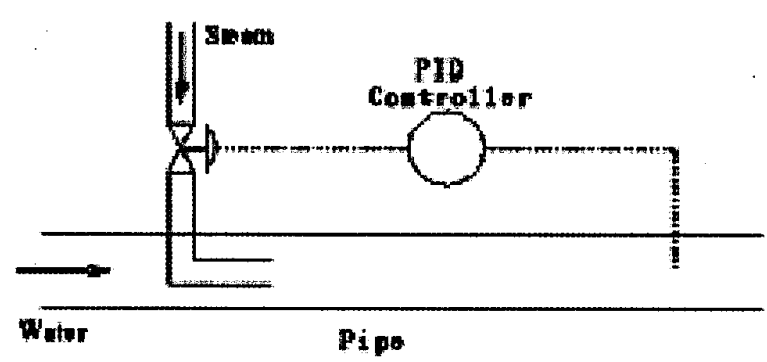

Figure 2: Water heater process

B. Simulation Design and Results Analysis

A LQG controller is designed for the water heater process as a benchmark controller using the cost function to specify the trade-off between regulation performance and cost of control.

$J(u)=\int_{0}^{\infty}\left(y^{2}+1.5 u^{2}\right) d t$

The LQ-optimal gain $K_{f}$ and estimator gain $K_{\text {est }}$ obtained via the Control Toolbox in the MATLAB are given by

$\left\{\begin{array}{l}K_{f}=0.2433 \\ K_{\text {est }}=6.7737\end{array}\right.$ 
The state estimator is represented by a matrix of transfer functions as follows.

$$
\left[\begin{array}{cc}
\frac{0.2965}{s+4.197} & \frac{0.5}{s+4.197} \\
\frac{4.017}{s+4.197} & \frac{6.774}{s+4.197}
\end{array}\right]
$$

Connecting the estimator and the optimal state-feedback gain, the final LQG controller gain is given by

$$
K(s)=-\frac{1.647}{s+4.319}
$$

The controller fault detection scheme shown in Figure 1 is implemented for the water heater to monitor the PID controller behaviors. At the instant 1000 , a designed fault is introduced into the actual controller by decreasing the I-gain value of the PID controller. The change of the PID controller leads process outputs moving away from the reference trajectory as shown in Figure 3. The outputs of the PID and LQG controller deviate from each other significantly shown in Figure 4 in the abnormal work state.

In the normal work state, 800 observed residuals are collected to fit the Gassian distribution parameters and the identified mean $\mu_{0}$ and variance $\sigma_{0}$ are -0.0081 and 0.0058 respectively. The fault detector uses a moving observation window to monitor the residuals and the threshold, $\lambda$, is set as 0.85 . The result of the likelihood ratio $s_{k}$ is shown in Figure 5. It is clear that from the instant 1000, the likelihood ratio increases significantly; it indicates the fault has taken place around the instant 1000.

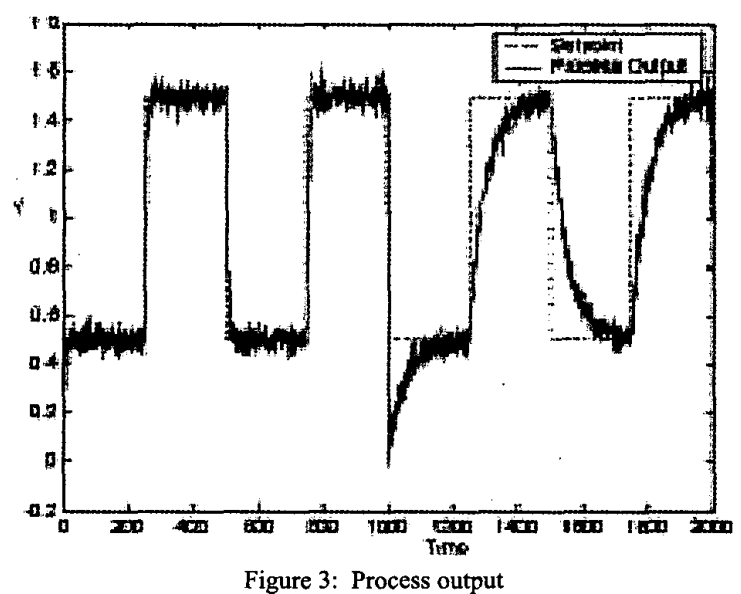

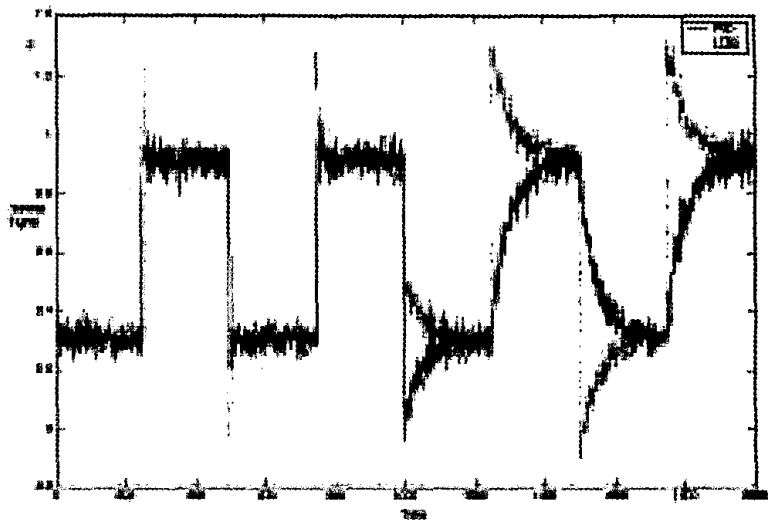

Figure 4: Control outputs of the PID and LQG controllers

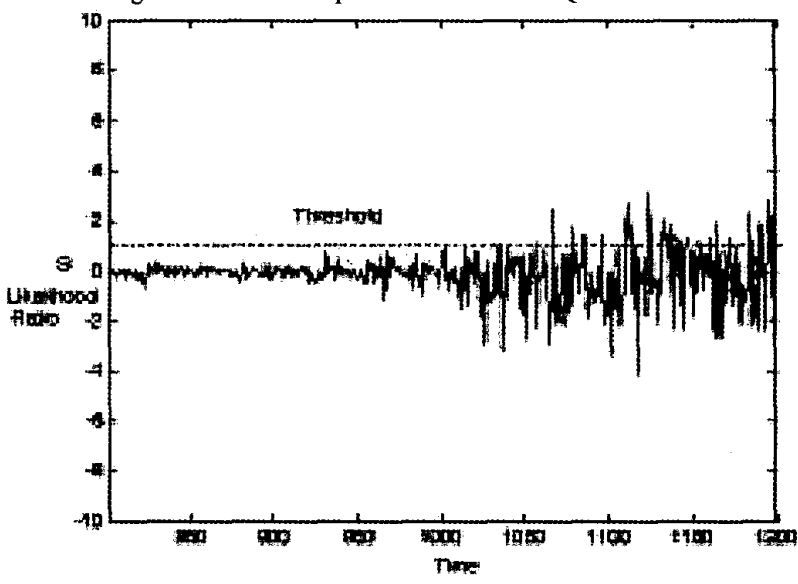

Figure 5: Likelihood ratio

\section{Conclusions}

Real-time monitoring and maintaining controller is an important issue in control system management. In this paper, we developed an online detection technique for control software monitoring. The detection is based on the analysis of the discrepancies of control signals of the actual controller and the benchmark controller, LQG. The residuals are assumed to be in an approximate Gassian distribution with a stable mean under normal operations, and a significant change in the mean under abnormal situations. The generalized likelihood ratio test is employed to online detect the change in the mean of the discrepancies between the actual and benchmark controllers. The proposed approach is implemented in a simulated water heater process with a PID controller. Simulation results demonstrate its validity and effectiveness. In general, this approach does not need any pre-knowledge about the actual control software or algorithm. Therefore it might be applied to any complex control systems.

\section{REFERENCES}

[1] M. King, B. Zhu, and S. Tang, "Optimal path planning," Mobile Robots, vol. 8, no. 2, pp. 520-531, March 2001.

[2] H. Simpson, Dumb Robots, 3rd ed., Springfield: UOS Press, 2004, pp.6-9. 
[3] M. King and B. Zhu, "Gaming strategies," in Path Planning to the West, vol. II, S. Tang and M. King, Eds. Xian: Jiaoda Press, 1998, pp. 158-176.

[4] B. Simpson, et al, "Title of paper goes here if known," unpublished.

[5] J.-G. Lu, "Title of paper with only the first word capitalized," J. Name Stand. Abbrev., in press.

[6] Y. Yorozu, M. Hirano, K. Oka, and Y. Tagawa, "Electron spectroscopy studies on magneto-optical media and plastic substrate interface," IEEE Translated J. Magn. Japan, vol. 2, pp. 740-741, August 1987 [Digest 9th Annual Conf. Magnetics Japan, p. 301, 1982].

[7] M. Young, The Technical Writer's Handbook, Mill Valley, CA: University Science, 1989. 